[CONTRIBUTION HROM THE KeNt Chemical Laboratory OF YaLe University.]

\title{
THE USE OF GALLIUM FERROCYANIDE IN ANALYSIS.
}

\author{
By Lyman E. Porter and Philip E. Browning. \\ Received October 30, 1920.
}

It has been shown ${ }^{1,2}$ that gallium may be separated from many elements by precipitation as the ferrocyanide from a solution containing as much as a third of its volume of conc. hydrochloric acid. Two problems arise in connection with the application of this reaction in analytical work, namely, filtration and the recovery of the gallium from the precipitated salt free from iron and the ferrocyanide radical. This paper gives the results of a study of these points.

The precipitate of gallium ferrocyanide is very gelatinous and, while it is possible to filter it on an ordinary paper filter, the filtration is very slow and it is difficult to wash the material. The large bulk of the precipitate and the slowness with which it settles make it impossible to decant the liquid with any success. The effect of changing the acid concentration and of precipitating the material in the presence of electrolytes such as potassium chloride and ammonium chloride was studied, but it was found that the precipitate does not settle any better under these conditions. Adding the reagent to the hot solution likewise failed to make any appreciable difference in the form of the precipitate and in its speed of filtration.

Various modifications of paper and asbestos filters were tested in an attempt to hasten the filtration. The use of asbestos or of paper on a Gooch crucible was found impossible, the precipitate passing through even a thick layer of the material, as it does likewise through paper on a funnel when suction is applied. If, however, a mat of fine filter paper fiber, best made by scratching some paper with a knife, is washed into the funnel, containing a double filter paper, the gallium ferrocyanide is held on it, provided the suction is not applied too strongly. If, as is often the case, the first portion of the filtrate is cloudy, it should be poured through the filter again, the resulting filtrate being clear. This process can be carried out in much less time than is required for the filtration of the same amount of material without the use of suction. Of 2 solutions, containing equal amounts of the gallium precipitate in $10 \mathrm{cc}$. of liquid, one required 15 minutes to be filtered once through a paper filter without suction, whereas the second, by the use of the method just described, was completely filtered in 2 minutes, including the second filtration of part of the solution. Table I shows the results of the quantitative determination of gallium as the ferrocyanide, the filtration having been

${ }^{1}$ Lecoq de Boisbaudran, Compt. rend., 94, 1154, 1228, 1439, 1628; 95, 157, 410, $503,1192,1332 ; 96,152,1696,1838 ; 97,142,295,522,623,730,1463$ (1882-1883).

"Browning and Porter, Am. J. Sci, 44, 221 (1917). 
made with suction. The material was ignited and weighed as the mixed oxides of gallium and iron, ${ }^{1}$ in the gallium ferrocyanide salt, $\mathrm{Ga}_{4}\left(\mathrm{FeC}_{6} \mathrm{~N}_{6}\right)_{3}$. If some of the iron remains as the carbide, as is probably the case, the resulting weight is not affected, on account of the identity of the molectlar weights of $\mathrm{Fe}_{2} \mathrm{O}_{3}$ and $2 \mathrm{FeC}_{2}{ }^{2}$

\begin{tabular}{|c|c|c|c|c|}
\hline \multicolumn{5}{|c|}{ TABLE I. } \\
\hline & $\begin{array}{l}\text { Gallium oxide } \\
\text { taken. } \\
\text { G. }\end{array}$ & $\begin{array}{l}\text { Mixed oxides } \\
\text { calculated. } \\
\text { G. }\end{array}$ & $\begin{array}{l}\text { Mixed oxides } \\
\text { found. } \\
\text { G. }\end{array}$ & $\begin{array}{c}\text { Error. } \\
\text { G. }\end{array}$ \\
\hline & 0.0236 & 0.0386 & 0.0400 & 0.0014 \\
\hline & 0.0236 & 0.0386 & 0.0395 & 0.0009 \\
\hline
\end{tabular}

This method of direct ignition is not specially recommended, as it gives high results which in small amounts of material show high percentage errors. It was, however, thought best to include it in the general study of the problem.

The recovery of gallium from the ferrocyanide by the ignition with ammonium nitrate and the subsequent separation of the gallium from iron by means of sodium hydroxide has been described by us. ${ }^{3}$ 'The possibility of recovering the gallium without the formation of ferric or ferrous salts and the necessity of separating it from these was studied. When the precipitated gallium ferrocyanide is treated with an alkali in solution it is decomposed into the soluble alkali salts of gallium and of ferrocyanide. It was found that when carbon dioxide is bubbled through such a solution to saturation the gallium is quantitatively precipitated as hydroxide, or basic carbonate, in a form easy to filter and readily washed free from ferrocyanide. This behavior is analogous to the reaction utilized in the detection of the constituents of zinc ferrocyanide. ${ }^{4}$ Table II

TABLE II.

\begin{tabular}{|c|c|c|c|}
\hline & $\begin{array}{c}\mathrm{Ga}_{2} \mathrm{O}_{3} \text { taken } \\
\mathrm{G} .\end{array}$ & $\begin{array}{c}\mathrm{Ga}_{2} \mathrm{O}_{3} \text { found. } \\
\mathrm{G} \text {. }\end{array}$ & $\underset{\text { Error }}{\text { Er. }}$ \\
\hline$\ldots$ & 0.0472 & 0.0460 & -0.0006 \\
\hline & 0.0236 & 0.0215 & -0.0021 \\
\hline & 0.0236 & 0.0225 & -0.0011 \\
\hline & 0.0236 & 0.0238 & +0.0002 \\
\hline & 0.0944 & 0.0951 & +0.0007 \\
\hline & 0.1038 & 0.1035 & -0.0003 \\
\hline
\end{tabular}

shows some results on the quantitative estimation of gallium after its precipitation as the ferrocyanide and the recovery of the gallium as described above. The ferrocyanide precipitate was in each case filtered on paper after standing for 2 days. In Expt. 1 the paper with the precipitate was treated directly in a beaker with sodium hydroxide solution and carbon dioxide, the precipitate and paper being filtered off. In Expt. 2 the precipitate was filtered and dissolved off the paper by sodium hydroxide solution and then treated with the carbon dioxide. In the

1 Compt. rend., 94, 1228 (1882).

2 Treadwell-Hall, "Analytical Chemistry," 4th Ed, I, 320.

${ }^{3}$ Browning and Porter, loc. cit.

"l'readwell-Hall, "Analytical Chemistry," 4t1, T;1, I, 319 
other cases the ferrocyanide was filtered on paper with suction in the presence of paper fiber, dissolved in sodium hydroxide solution and finally precipitated by carbon dioxide from a volume of $100 \mathrm{cc}$.

When an alkaline solution of gallium ferrocyanide is boiled with ammonium chloride a precipitate of gallium is formed, which consists chiefly of the ferrocyanide. If, however, the ferrocyanide is oxidized over to the ferricyanide by hydrogen peroxide in alkaline solution before the ammonium chloride treatment, the precipitate obtained is gallium hydroxide free from any cyanide radicals, as shown by qualitative tests. 'The oxidation may be made with a nitrate, but the peroxide method is the more satisfactory. 'This method for the recovery of gallium from the ferrocyanide works well and is rather quicker than the carbon dioxide method. Quantitative results are given in Table III of some determinations by this method in which the gallium was precipitated as the ferrocyanide and dissolved directly in sodium hydroxide without previous filtration.

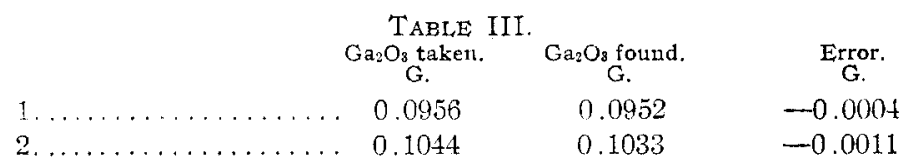

Of the elements commonly associated with gallium, whose salts are soluble in alkali, zinc is the most common one that forms an insoluble ierrocyanide. The insolubility of zinc ferricyanide in acid and in ammonium hydroxide makes the separation of gallium from the ferrocyanide by means of the peroxide method impossible if zinc is present. In such a case, however, either of the following 2 methods may be employed.

In the first case the mixed ferrocyanides are dissolved in sodium hydroxide solution and the bases precipitated together by carbon dioxide. The gallium may now be separated from the zinc by dissolving the precipitate in hydrochloric acid and boiling the solution with ammonium acid sulfite to precipitate the gallium. ${ }^{1}$ The following procedure may also be used. The alkali solution of the ferrocyanides is treated with hydrogen sulfide to precipitate the zinc, ${ }^{2}$ which even in the presence of the ferrocyanide is precipitated as the sulfide. From the filtrate the gallium may be recovered free from ferrocyanide either by precipitation with carbon dioxide, or by boiling with ammonium chloride after the oxidation of the excess of sulfide and of the ferrocyanide by hydrogen peroxide.

\section{Summary.}

1. I method of filtering gallium ferrocyanide with suction has been described.

2. Methods for the recovery of gallium from its ferrocyanide salt by alkali and carbon dioxide, by sodium hydroxide, and by hydrogen peroxide and ammonium chloride have been developed.

1 Forter and Browning, This Journal, 4I, 1491 (1919).

${ }^{2}$ Hrowning and Porter, loc. cit. 
3. Methods for the recovery of gallium from its ferrocyanide in the presence of zinc have been described.

New Haven, Cons.

[CONTRIBUTION FROM THE KENT CHEMICAL LABORATORY OF YALE UNIVERSITY.]

\section{THE QUALITATIVE SEPARATION AND DETECTION OF URANIUM, VANADIUM AND CHROMIUM WHEN PRESENT TOGETHER.}

By Philip E. Browning.

Received October 30, 1920.

A. A. Noyes and his associates in their excellent treatise on a System of Qualitative Analysis, which includes some of the less common elements, give the following procedure for the separation of uranium, vanadium and chromium when present together. ${ }^{1}$ To the solution containing sodium chromate, sodium vanadate, and the double sodium-uranium carbonate nitric acid is first added to neutrality and then in slight excess and the chromium is precipitated as lead chromate by lead nitrate. After the removal of the lead chromate by filtration the excess of lead is removed by hydrogen sulfide and the lead sulfide filtered off. The excess of hydrogen sulfide is removed by boiling, re-oxidation effected by bromine, the excess of bromine removed by boiling, and after acidification with acetic acid, the uranium is precipitated by sodium phosphate. The presence of uranium is confirmed by dissolving the precipitate in hydrochloric acid, adding sodium chloride and treating with potassium ferrocyanide. The filtrate from the uranium phosphate is made alkaline with ammonium hydroxide and saturated with hydrogen sulfide, a pink or violet color indicating oxysulfide of vanadium, if that element is present.

The work to be described is a modification of the above method involving the use of another reaction and obviating the necessity of the introduction and subsequent removal of lead.

The solution containing chromic and vanadic acids together with a uranium salt is made faintly alkaline with ammonia and then faintly acid with acetic acid and treated with ammonium phosphate. This precipitates the phosphate of uranium which is filtered off and washed. If the filtration is difficult it may be successfully accomplished by the introduction of some shredded filter paper. The precipitate on the filter paper is treated with an acidified solution of potassium ferrocyanide and if uranium is present a characteristic red-brown stain is the result. The filtrate is then treated with sulfurous acid in distinct excess, which reduces the chromic and vanadic acids and the excess of the sulfurous acid is removed by boiling. This solution is then treated with bromine water in distinct excess and the excess of bromine is removed by boiling. The bromine oxidizes the vanadium to the acid condition and does not affect

\footnotetext{
1 'This Jolrnai, 3o, 481 (1908).
} 